\title{
NONLINEAR ACTUATOR MODEL FOR FLEXOELECTRIC MEMBRANES
}

\author{
A.D. REY \\ Department of Chemical Engineering, McGill University, Montreal, Quebec, Canada.
}

\begin{abstract}
Synthetic and biological flexoelectric membranes are actuators that bend under the action of external electric fields, a phenomenon of interest to the development of emerging adaptive materials as well as biological mechano-transduction. This paper presents an actuator model of flexoelectric membranes based on a Helmholtz free energy that incorporates tension, bending, and torsion as well as polarization and dielectric energies. The electro-elastic components of the membrane tension, moment tensor and tensor are derived and used to construct an actuator model that includes dissipation due to viscous fluids in contact with the membrane. The actuator model is expressed by a balance between the externally imposed electric forces, the viscous dissipation of the contacting fluid phases, and the elastic storage of the membrane. The nonlinearity is shown to originate in the viscous dissipation. The model is analyzed for externally imposed oscillating electric fields. The Deborah number De given by the ratio of driving frequency and the resonant frequency is shown to control the viscoelastic response. The key findings are: (i) for De $\ll 1$ the response is purely elastic and the electric energy is stored in the elastic deformations of the membrane; (ii) at larger De, the response is anharmonic and viscoelastic; (iii) due to the nature of the viscous nonlinearity only even harmonics are generated in the response; and (iv) secondary resonant frequencies appear at lower driving frequencies. These finding contribute towards the emerging understanding of flexoeletricity in biological membranes, pioneered by Petrov and co-workers (Petrov, A.G., The Lyotropic State of Matter, Gordon and Breach Science Publishers: Amsterdam, 1999).

Keywords: actuator model, electro-elastic response, flexoelectric membranes, frequency response.
\end{abstract}

\section{INTRODUCTION}

Current interest in synthetic and biological functional materials with sensor and actuator abilities is driven by the need for advanced performance in aerospace, transportation, drug delivery, and medical devices [1]. The sought after materials have the ability to change shape under external fields (actuator mode) and generate a detectable signal due to mechanical deformation (sensor mode). An additional motivation is the understanding of many biological processes that involves mechanotransduction mechanisms, which also involve couplings between mechanical-chemical-electrical fields. Materials displaying sensor/actuator responses are known as adaptive materials. Examples include piezoelectrics, shape memory alloys, electro-magnetorheological fluids, liquid crystals, and polymer gels. Synthetic and biological membranes that display polarization under bending deformations are another but less explored class of adaptive materials known as flexoelectric [2]. This paper focuses only on flexoelectric membranes.

Flexoelectricity is the property of synthetic and biological flat membranes to bend under the imposition of an external electric field, and the capacity to become polarized under bending [2]. The former is known as the actuator mode and the latter the sensor mode [2]. The basic science and applications of membrane flexoelectricity was developed by Petrov and co-workers and is described in detail in [2-4]. Figure 1 shows the sensor (full line) and actuator (dashed line) modes of flexoelectric membranes.

The flexoelectric actuator mode is given by a linear relation between input (electric field E) and out-put (average curvature $\mathrm{H}$ ):

$$
2 \mathrm{H}=\operatorname{trace} \mathbf{b}=\frac{\mathbb{C}}{\mathrm{k}_{\mathrm{c}}} \mathrm{E}
$$




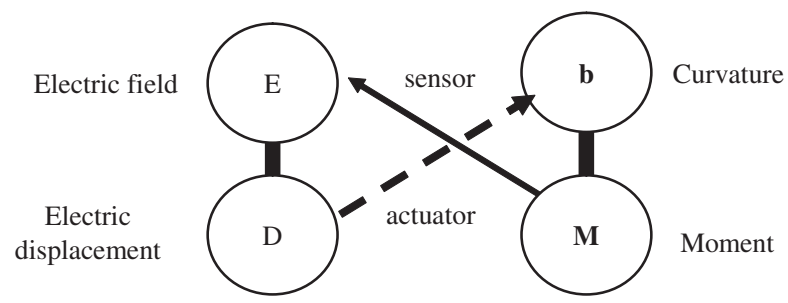

Figure 1: Schematic of sensor and actuator modes in flexoelectric membranes.

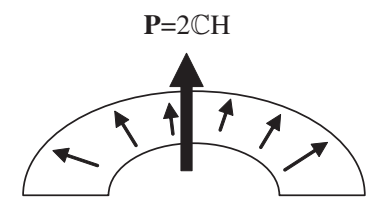

sensor mode

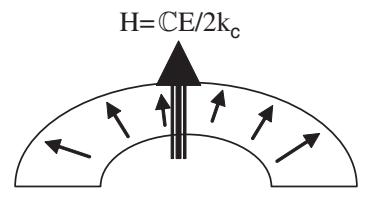

actuator

Figure 2: Schematics of sensor and actuator modes of flexoelectric membranes.

where $H=\mathbf{I}_{\mathrm{s}}: \mathbf{b} / 2, \mathbf{b}=-\nabla_{\mathrm{s}} \mathbf{k}$ is the symmetric curvature tensor $[5,6], \mathbf{k}$ is the membrane unit normal, $\nabla_{\mathrm{s}}(\bullet)=\mathbf{I}_{\mathrm{s}} \cdot \nabla(\bullet)$ is the surface gradient operator $[5,6], \mathbf{I}_{\mathrm{s}}=\mathbf{I}-\mathbf{k k}$ is the surface unit tensor, $\mathbb{C}$ is the flexoelectric constant [2], $\mathrm{k}_{\mathrm{c}}$ is the membrane bending rigidity [2], and $\mathrm{E}$ is the magnitude of the imposed electric field. Typical values of $\mathbb{C}$ for dipolar lipid membranes are $10^{-20} \mathrm{C}[2]$. The flexoelectric sensor mode of synthetic and biological membranes is given by a linear relation between input (average curvature $H$ ) and output (electric polarization $\mathbf{P}$ ):

$$
\mathbf{P}=\mathbb{C} \mathbf{k}\left(\mathbf{I}_{\mathbf{s}}: \mathbf{b}\right)=\mathbb{C} \mathbf{k}(2 \mathrm{H})
$$

where $\mathbf{P}$ is aligned along the membrane unit normal $\mathbf{k}$. Membrane bending distortions hence create an electric polarization. Figure 2 shows the schematics of the sensor and actuator modes that explain the physical meaning of eqns. (1) and (2).

The relationship between displacement $\mathbf{D}$, polarization $\mathbf{P}$ and electric field $\mathbf{E}$ appearing in Fig. 1 is:

$$
\mathbf{D}=4 \pi \mathbf{P}+\varepsilon \cdot \mathbf{E}
$$

The relationship between the moment tensor $\mathbf{M}$ and the curvature tensor $\mathbf{b}$ is defined in eqn. (6). The main difference between flexoelectric and piezoelectric effects is that in the latter, polarization is coupled to the membrane strain, while in the former it is coupled to curvature. The implications of this fundamental difference between piezo- and flexoelectric membranes in shape changing abilities under externally imposed electric fields were discussed in [7, 8].

As demonstrated by Petrov [2], flexoelectricity is important in a number of biophysical processes including protein-protein interaction in curved membranes, ion transport, membrane fluctuations, mechano-transduction, biomolecular electronics, and functioning of outer hair cells in hearing [2]. In many biological processes, the membrane is in contact with viscous and/or viscoelastic phases and the fields are dynamic. Laboratory characterization of flexoelectricity also is carried-out using oscillatory electric fields [2]. Hence, a better understanding of the frequency response of flexoelectric membranes to oscillating electric fields is necessary for a better understanding of biological 
processes as well as for the characterization of membrane electro-mechanics. Previous analysis and experiments show that when the membrane is immersed in viscous media, the response to oscillating electric fields becomes nonlinear as sufficiently high driving frequencies [2]. This observation has a number of potential implications, including anharmonic frequency response, multiple sub-harmonic resonances, and combination frequencies when two fields of different frequencies are applied [9]. Future experimental work in conjunction with theory and simulation is necessary to establish the impact of these effects on biological processes.

This paper contributes to the on-going efforts in membrane mechano-transduction and electromechanics by developing a simple model that predicts the frequency response of a flexoelectric membrane to oscillating electric fields in the presence of contacting viscous fluids. The response of biological cells to oscillating electric fields is of on-going interest because it affects cell function [10, 11]. This paper builds on a previously presented static model for flexoelectric membranes under constant fields and immersed in inviscid fluids $[8,9]$.

The objectives of this paper are:

1. to derive a transient membrane flexoelectric model in the presence of viscous dissipation and fluctuating electric fields;

2. to identify the main features of the frequency response and correlate them to the electromechanical properties of flexoelectric membranes.

To avoid repetition of lengthy derivations, the reader is refereed to [7, 8] for mathematical details of the static flexoelectric membrane model. In this paper, we will only focus on derivations involving viscous dissipation and fluctuating electric fields.

\section{MEMBRANE ELECTRODYNAMICS}

The description of membrane electro-thermodynamics is based on the expression of the membrane stress tensor $\mathbf{T}_{\mathbf{m}}$ in terms of the bending moment tensor $\mathbf{M}$ and the tangential electro-capillary vector $\mathbb{Q}_{/ /}$. The bending moment tensor $\mathbf{M}$ is a symmetric $2 \times 2$ tensor that measures changes in free energy due to changes in the $2 \times 2$ symmetric tangential membrane curvature tensor $\mathbf{b}$. The electro-capillary vector is a tangential vector that relates free energy changes to changes in the membrane unit normal $\mathbf{k}$. To derive the membrane stress tensor, one starts with the Helmholtz free energy and then the stress and moment tensors are obtained through a variational calculation.

The Helmholtz free energy per unit mass $\hat{A}$ of the membrane is given by [7, 8]:

$$
\hat{\mathrm{A}}=\mu+\frac{\gamma}{\rho}-\frac{\mathbf{P} \cdot \mathbf{E}}{\rho}-\frac{\boldsymbol{\varepsilon}: \mathbf{E E}}{8 \pi \rho}
$$

where $\mu$ is the Gibbs function per unit mass, $\rho$ is the surface mass density, $\gamma$ is the membrane tension, and $\varepsilon$ is the dielectric tensor. In the absence of an electric field, we recuperate the surface Euler equation [6]. At a constant electric field $\mathbf{E}$, the total differential of $\hat{A}$ is [7, 8]:

$$
\mathrm{d} \hat{\mathrm{A}}=-\left(\gamma-\mathbf{P} \cdot \mathbf{E}-\frac{\boldsymbol{\varepsilon}: \mathbf{E E}}{8 \pi}\right) \frac{\mathrm{d} \rho}{\rho^{2}}+\frac{\mathbf{M}}{\rho}: \mathrm{d} \mathbf{b}+\frac{\mathbb{Q}_{/ /}}{\rho} \cdot \mathrm{d} \mathbf{k}
$$

The membrane tension $\gamma$, bending moment tensor $\mathbf{M}$, and the electro-capillary vector $\mathbb{Q}_{/ /}$are $[7,8]$ :

$$
\gamma=-\rho^{2} \frac{\partial \hat{\mathrm{A}}}{\partial \rho}+\mathbf{P} \cdot \mathbf{E}+\frac{\boldsymbol{\varepsilon}: \mathbf{E E}}{8 \pi}, \quad \mathbf{M}=\mathbf{M}^{\mathrm{T}}=\left(\rho \frac{\partial \hat{\mathrm{A}}}{\partial \mathbf{b}}\right)_{\rho, \mathbf{k}}, \quad \mathbb{Q}_{/ /}=\left(\rho \mathbf{I}_{\mathrm{s}} \cdot \frac{\partial \hat{\mathrm{A}}}{\partial \mathbf{k}}\right)_{\rho, \mathbf{b}}
$$


where the superscript "T" in eqn. (6b) denotes transpose. Equation (6) shows that $(\gamma-\mathbf{P} \cdot \mathbf{E}-\boldsymbol{\varepsilon}: \mathbf{E E} / 8 \pi) / \rho^{2}$ is the conjugate to $\rho, \mathbf{M} / \rho$ is the conjugate of curvature $\mathbf{b}$, and the local electro-capillary vector $\mathbb{Q}_{/ /} / \rho$ is the conjugate to $\mathbf{k}$. Using eqn. (4), a direct variational calculation gives the electro-elastic membrane tensor $\mathbf{T}_{\mathrm{m}}[7,8]$ :

$$
\mathbf{T}_{\mathrm{m}}=\left(\gamma-\mathbf{P} \cdot \mathbf{E}-\frac{\boldsymbol{\varepsilon}: \mathbf{E} \mathbf{E}}{8 \pi}-\frac{1}{2}(\mathbf{M}: \mathbf{b})\right) \mathbf{I}_{\mathrm{s}}-\overparen{\mathbf{M} \cdot \mathbf{b}}-\left(\mathbb{Q}_{/ /}+\left(\nabla_{\mathrm{s}} \cdot \mathbf{M}\right)\right) \mathbf{k}
$$

where the overbar on the second term $(\overparen{\mathbf{M} \cdot \mathbf{b}})$ denotes a traceless tensor. The first term in eqn. $(7)$ are the extension stresses $\left(\mathbf{T}^{\text {exten}}\right)$, the second the shear stresses $\left(\mathbf{T}^{\text {shear }}\right)$, and the third the bending stresses ( $\mathbf{T}^{\text {bend }}$ ). Figure 3 shows the membrane stress components (left) acting on a patch of the membrane and the matrix (right) representation. Extension and shear stresses do not involve bending. Bending stresses are associated with shape changes and act in a direction normal to the membrane.

\section{CONSTITUTIVE EQUATIONS FOR ELECTRO-ELASTIC MEMBRANES}

The Helfrich free energy per unit area $\rho \hat{A}_{c}$ [12], widely used to describe the elasticity of membranes and surfactant-laden interfaces, reads:

$$
\rho \hat{\mathrm{A}}_{\mathrm{c}}(\mathrm{H}, \mathrm{K})=2 \mathrm{k}_{\mathrm{c}}(\mathrm{H})^{2}+\overline{\mathrm{k}}_{\mathrm{c}} \mathrm{K}
$$

where $\mathrm{K}$ is the Gaussian curvature and $\overline{\mathrm{k}}_{\mathrm{c}}$ is the torsion elastic moduli. Bending and torsion deformation in membranes are indicated in Fig. 4.

Under an external electric field E, eqns. (2-4) and (8) show that the total Helmholtz free energy per unit area $\rho \hat{A}$ is $[7,8]$ :

$$
\rho \hat{\mathrm{A}}(\mathrm{H}, \mathrm{K})=2 \mathrm{k}_{\mathrm{c}}(\mathrm{H})^{2}+\overline{\mathrm{k}}_{\mathrm{c}} \mathrm{K}-2 \mathbb{C}(\mathbf{k} \cdot \mathbf{E}) \mathrm{H}-\frac{\varepsilon: \mathbf{E E}}{8 \pi}
$$
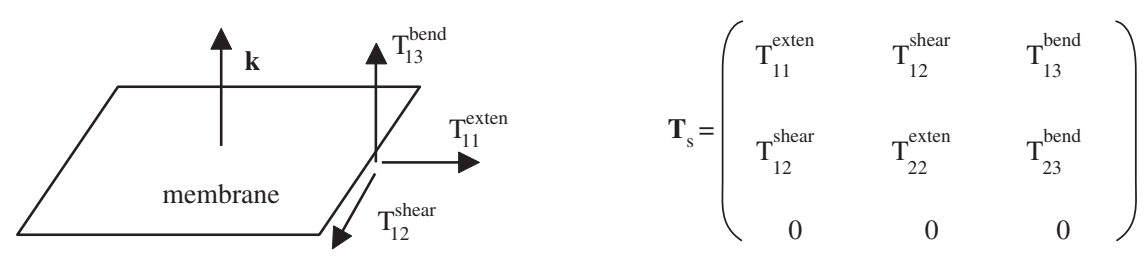

Figure 3: Schematic of stresses acting on a membrane (left) and matrix representation (right).
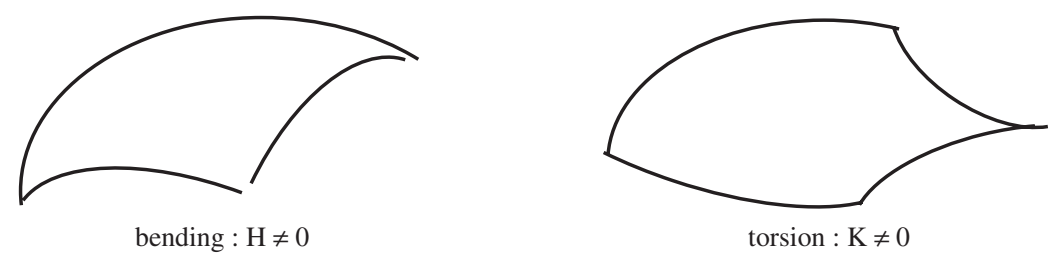

Figure 4: Schematic of bending (left) and torsion (right) deformation in membranes. 
To derive an expression of the actuator model for a flexoelectric membrane using eqn. (9), we must find expressions for the following primary quantities: (i) membrane tension $\gamma$, (ii) bending moment tensor $\mathbf{M}$, and (iii) the membrane capillary pressure $\mathbf{T}_{\mathrm{s}} \mathbf{:} \mathbf{b}$, as follows. Using eqns. (4) and (9), the membrane tension $\gamma$ is found to be $[7,8]$ :

$$
\gamma=\gamma_{\mathrm{o}}+\mathbb{C H}(\mathbf{k} \cdot \mathbf{E})+\frac{1}{2} \mathbf{M}: \mathbf{b}
$$

where $\gamma_{\mathrm{o}}$ is the tension at zero field $(\mathbf{E}=\mathbf{0})$ and zero curvature $(\mathrm{H}=\mathrm{K}=0)$. Expressing the symmetric $2 \times 2$ moment tensor $\mathbf{M}$ in terms of unit tensor $\mathbf{I}_{\mathrm{s}}$ and curvature tensor $\mathbf{b}$ we find using eqns. (6) and (9) that $[7,8]$ :

$$
\mathbf{M}=\frac{\rho \partial \hat{\mathrm{A}}}{\partial \mathbf{b}}=\left(\frac{\mathrm{C}_{1}}{2}+2 \mathrm{C}_{2} \mathrm{H}\right) \mathbf{I}_{\mathrm{s}}-\mathrm{C}_{2} \mathbf{b}
$$

where the two bending coefficients $\left\{\mathrm{C}_{1}, \mathrm{C}_{2}\right\}$ are $[7,8]$ :

$$
\mathrm{C}_{1}=4 \mathrm{k}_{\mathrm{c}}(\mathrm{H})-2 \mathbb{C}(\mathbf{k} \cdot \mathbf{E}), \quad \mathrm{C}_{2}=\overline{\mathrm{k}}_{\mathrm{c}}
$$

Lastly, using eqns. (7), (10), and (11) the total membrane capillary pressure $\mathbf{T}_{\mathrm{s}}: \mathbf{b}$ is $[7,8]$ :

$$
\mathbf{T}_{\mathrm{m}}: \mathbf{b}=\left(\gamma_{\mathrm{o}}-\frac{\boldsymbol{\varepsilon}: \mathbf{E E}}{8 \pi}\right) 2 \mathrm{H}
$$

Equations (11) and (12) show the origin of the actuator mode in flexoelectric membranes, since the electric field has the ability to create a contribution $-\mathbb{C}(\mathbf{k} \cdot \mathbf{E}) \mathbf{I}_{\mathrm{s}}$ to the moment tensor $\mathbf{M}$. Neglecting torsion $\left(\mathrm{C}_{2}=\overline{\mathrm{k}}_{\mathrm{c}}=0\right)$ it is seen that when $\mathbf{M}=\mathbf{0}$, eqn. (11) implies eqn. (1). Equation (13) indicates that the capillary pressure $\mathbf{T}_{\mathrm{s}}: \mathbf{b}$ is a function of the dielectric energy.

\section{NONLINEAR ACTUATOR MODEL FOR FLEXOELECTRIC MEMBRANES}

In this section, we use eqns. (11) and (13) to derive the actuator model flexoelectric membrane. The actuator model is found by using an integral normal forces balance equation on a membrane, subjected to moments $(\mathbf{M})$ along its edges, normal forces (kk : $\Delta \mathbf{T}_{\mathrm{b}}$ ) due to contacting viscous phases, and resisting capillary forces $\left(\mathbf{T}_{\mathrm{m}}: \mathbf{b}\right)$ due to tension and dielectric energy and assuming constant curvature deformations. In this paper, we assume that deformations are always spherical and the average curvature is $H=-1 / R$, where $\mathrm{R}$ is the sphere radius.

To construct a tractable actuator model for flexoelectric membranes we use the integral balance approach widely used in interfacial transport phenomena. The virtual power expression due to a normal displacement $\xi_{\perp}$ of the membrane reads [7,8]:

$$
\int\left\{\mathbf{T}_{\mathrm{m}}: \mathbf{b}+\mathbf{k k}: \Delta \mathbf{T}_{\mathrm{b}}\right\} \xi_{\perp} \mathrm{d} S+\oint_{\mathrm{C}} \boldsymbol{\mu} \cdot \mathbf{M} \cdot\left(\nabla_{\mathrm{s}} \xi_{\perp}\right) \mathrm{d} \ell=0
$$

where the first integral denotes normal forces over the surface $\mathrm{S}$, and the second is the normal force due to couples acting on the edge $\mathrm{C}$ of the membrane; $\boldsymbol{\mu}$ is the tangent to the membrane at the membrane edge C. Due to the assumed constant curvature the integrands in eqn. (14) are constants and hence:

$$
\left\{\mathbf{T}_{\mathrm{m}}: \mathbf{b}+\mathbf{k k}: \Delta \mathbf{T}_{\mathrm{b}}\right\} \int \xi_{\perp} \mathrm{d} S+\boldsymbol{\mu} \cdot \mathbf{M} \cdot \oint_{\mathrm{C}}\left(\nabla_{\mathrm{s}} \xi_{\perp}\right) \mathrm{d} \ell=0
$$


We now define a geometric factor that collects all the geometric information appearing in eqn. (15):

$$
\mathfrak{I}=\frac{\oint_{\mathrm{C}}\left|\nabla_{\mathrm{S}} \xi_{\perp}\right| \mathrm{d} \ell}{\int \xi_{\perp} \mathrm{dS}}=\frac{\left|\nabla_{\mathrm{s}} \xi_{\perp}\right| \oint_{\mathrm{C}} \mathrm{d} \ell}{\int \xi_{\perp} \mathrm{dS}}=\frac{\text { membrane slope } \times \text { edge length }}{\text { enclosed volume }}
$$

The unit of the geometric factor $\mathfrak{I}$ is reciprocal area. Introducing the shape factor $\mathfrak{I}$ into the integral shape equation (eqn. (15)) gives:

$$
\mathbf{k k}: \Delta \mathbf{T}_{\mathrm{b}}+\mathbf{T}_{\mathrm{m}}: \mathbf{b}+(\boldsymbol{\mu} \cdot \mathbf{M} \cdot \boldsymbol{\mu}) \mathfrak{I}=0
$$

Using eqns. (11)-(13), neglecting dielectricity, the spherical electro-viscoelastic shape equation is:

$$
\underbrace{\mathbb{C E E} \mathfrak{I}}_{\text {driving force }}=\underbrace{\mathbf{k k}: \Delta \mathbf{T}_{\mathrm{b}}}_{\text {viscous bulk }}+\underbrace{2 \mathrm{H}\left(\gamma_{\mathrm{o}}+\left(2 \mathrm{k}_{\mathrm{c}}+\overline{\mathrm{k}}_{\mathrm{c}}\right) \frac{\mathfrak{I}}{2}\right)}_{\text {membrane elasticity }}
$$

where the imposed field $\mathrm{E}=\mathbf{E} \cdot \mathbf{k}$ is the driving force which balanced by the viscous dissipation in the bulk fluid phases and the elastic deformation of the membrane. Equation (18) is the actuator model for a flexoelectric membrane of constant curvature in the absence of dielectricity. In the absence of viscous bulk phases contacting the membrane, and neglecting tension and torsion $\left(\gamma_{\mathrm{o}}=\overline{\mathrm{k}}_{\mathrm{c}}=0\right)$ eqn. (18) gives eqn. (1). Equation (18) indicates that the electric energy pumped into the actuator system is partly dissipated in the contacting fluid phases and partly stored in the elastic membrane. To model a specific actuator system, the jump stresses must be expressed in terms of the average curvature $\mathrm{H}$ and its time derivative $\mathrm{dH} / \mathrm{dt}$.

\section{OSCILLATING MEMBRANE UNDER CAPILLARY CONFINEMENT}

A geometry that is used in the experimental characterization of membrane flexoelectricity is the capillary tube [2], where the membrane is attached to the wall of the capillary, as shown in Fig. 5a. The tube is filled with a fluid of viscosity $\eta$ and the oscillating electric field $E(t)$ creates a periodic response in the membrane (see Fig. 5b).

Using an oscillating electric field distorts the membrane into a spherical cusp of height $h(t)$, and radius $\mathrm{R}(\mathrm{t})$, shown in Fig. 6 . In the spherical cusp geometry, and with a capillary of radius "a," the geometric factor in eqn. (16) is $\mathfrak{I}=8 / \mathrm{a}^{2}$ and the volume of the spherical cusp is $\pi \mathrm{a}^{2} \mathrm{~h} / 2$.

As the membrane oscillates due to the imposed oscillating electric field $E(t)$, a viscous flow is created in the adjoining viscous fluid phases (see Fig. 5a), which produces the viscous force (bulk stress jump) kk : $\Delta \mathbf{T}_{\mathrm{b}}$. Following [2], we assume that the viscous flow is described by incompressible Poiseuille flow [13]. The geometry and set-up is closely related to that used in pressure transduction systems [14]. Using cylindrical coordinates $(r, \phi, z)$, the velocity field $\mathbf{v}$ in the viscous fluid phase reads: $\mathbf{v}=\left(0,0, \mathrm{v}_{\mathrm{z}}(\mathrm{r}, \mathrm{t})\right)$, where $\partial \mathrm{v}_{\mathrm{z}}(\mathrm{r}, \mathrm{t}) / \partial \mathrm{z}=0$. The solution to the Navier-Stokes equations in the absence of inertia leads to the well-known formulas for the axial velocity $\mathrm{v}_{\mathrm{z}}(\mathrm{r}, \mathrm{t})$, average axial velocity $\left\langle\mathrm{v}_{\mathrm{z}}\right\rangle$ and volumetric flow rate $\mathrm{Q}(\mathrm{t})$ :

$$
\mathrm{v}_{\mathrm{z}}(\mathrm{r}, \mathrm{t})=2\left\langle\mathrm{v}_{\mathrm{z}}\right\rangle\left(1-\left(\frac{\mathrm{r}}{\mathrm{a}}\right)^{2}\right) ; \quad\left\langle\mathrm{v}_{\mathrm{z}}\right\rangle=-\frac{\mathrm{a}^{2}}{4 \eta} \frac{\partial \mathrm{p}}{\partial \mathrm{z}} ; \quad \mathrm{Q}(\mathrm{t})=\left\langle\mathrm{v}_{\mathrm{z}}\right\rangle \frac{\pi \mathrm{a}^{2}}{2}=-\frac{\pi \mathrm{a}^{4}}{8 \eta} \frac{\partial \mathrm{p}}{\partial \mathrm{z}}
$$

Under the condition $\mathrm{h} / \mathrm{a}=1$, the volume of the spherical cap is: $\mathrm{V}(\mathrm{t})=\pi \mathrm{a}^{2} \mathrm{~h}(\mathrm{t}) / 2$. To find the flow rate $\mathrm{Q}$ in terms of the curvature dynamics we first consider flow rate in terms of the time 


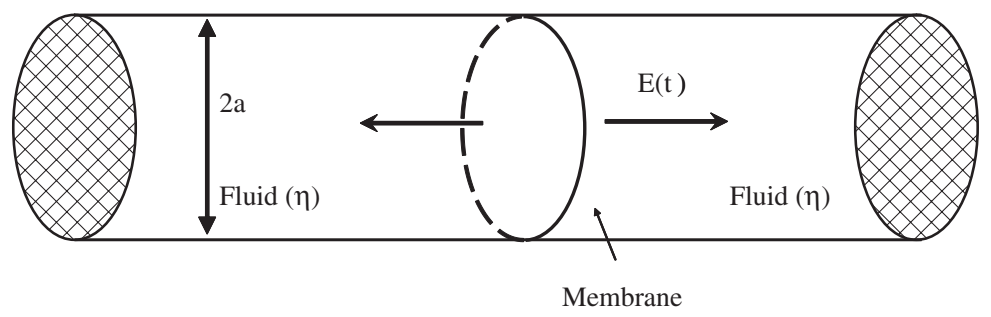

(a)
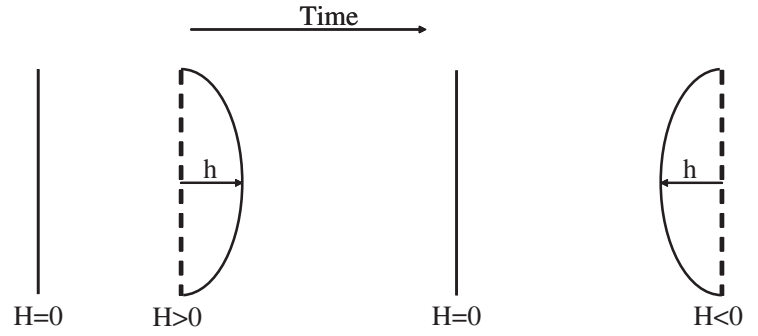

(b)

Figure 5: (a) Schematic of a flexoelectric membrane anchored in a capillary of radius "a" containing a fluid of viscosity $\eta$. As the field $E(t)$ oscillates, it creates distortions in the membrane (b) indicated by a nonzero average curvature $\mathrm{H}$.

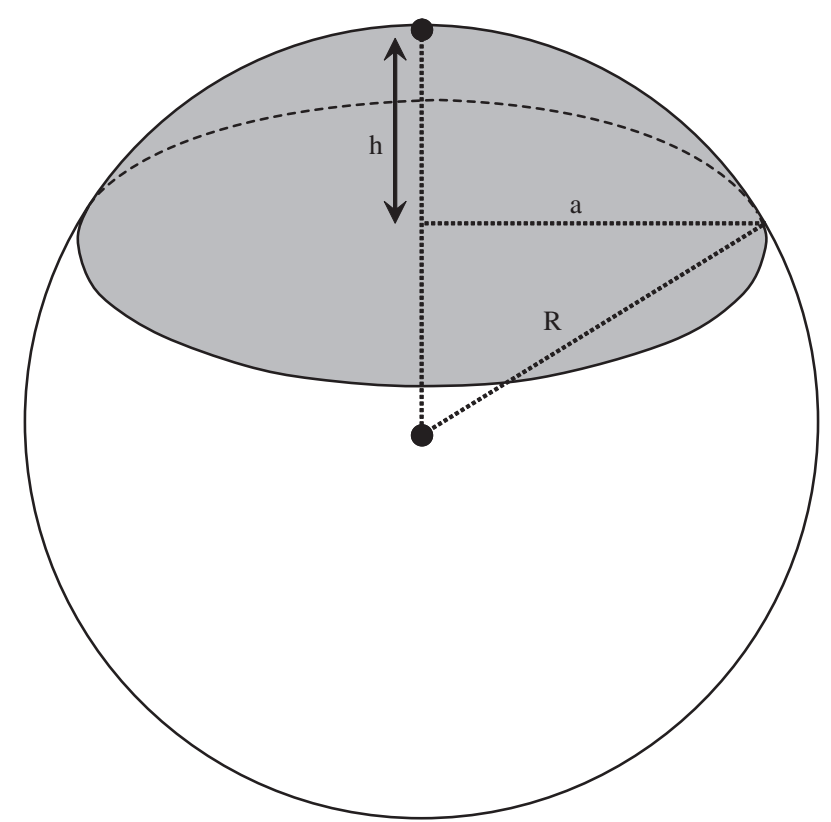

Figure 6: Schematic of the spherical cusp (shaded area) shape adopted by the flexoelectric membrane when subjected to the electric field E. 
derivative of the volume, $2 \mathrm{dV} / \mathrm{dt}=\mathrm{Q}$, which upon use of eqn. (19c) gives the relation between $\mathrm{dh} / \mathrm{dt}$ and $\left\langle\mathrm{v}_{\mathrm{z}}\right\rangle$ :

$$
\frac{\mathrm{dh}}{\mathrm{dt}}=\frac{\left\langle\mathrm{v}_{\mathrm{z}}\right\rangle}{2}
$$

Using the geometric relation for the spherical cusp $\mathrm{a}^{2}=-2 \mathrm{~h} / \mathrm{H}$ and eqns. (19c) and (20), the flow rate can be expressed in terms of $\mathrm{dH} / \mathrm{dt}$ :

$$
\mathrm{Q}(\mathrm{t})=\frac{\mathrm{dH}}{\mathrm{dt}} \frac{\pi \mathrm{a}^{4}}{2}
$$

Using eqns. (19b), (19c), and (21) we find the time rate of change of curvature as a function of the pressure drop:

$$
\frac{\mathrm{dH}}{\mathrm{dt}}=-\frac{1}{2 \eta} \frac{\partial \mathrm{p}}{\partial \mathrm{z}}=\frac{1}{4 \eta} \frac{\Delta \mathrm{P}}{\mathrm{h}}
$$

where following [2] we used $-2 \partial \mathrm{p} / \partial \mathrm{z}=\Delta \mathrm{P} / \mathrm{h}$. Using the relation $\mathrm{a}^{2}=-2 \mathrm{~h} / \mathrm{H}$ and eqn. (22) we find the stress jump:

$$
\mathbf{k k}: \Delta \mathbf{T}_{\mathrm{b}}=\Delta \mathrm{P}=2 \eta \mathrm{a}^{2} \mathrm{H} \frac{\mathrm{dH}}{\mathrm{dt}}
$$

Replacing the stress jump into eqn. (18) gives the nonlinear ordinary differential equation for $\mathrm{H}(\mathrm{t})$ :

$$
\mathrm{E}(\mathrm{t})=\left\{\frac{2 \eta \mathrm{a}^{2} \mathrm{H}}{\mathbb{C} \mathfrak{I}}\right\} \frac{\mathrm{dH}}{\mathrm{dt}}+\left\{\frac{2}{\mathbb{C} \mathfrak{I}}\left(\gamma_{\mathrm{o}}+\left(2 \mathrm{k}_{\mathrm{c}}+\overline{\mathrm{k}}_{\mathrm{c}}\right) \frac{\mathbb{C}}{2}\right)\right\} \mathrm{H}
$$

The nonlinearity $\mathrm{HdH} / \mathrm{dt}$ in eqn. (24) appears in the viscous dissipation term and arises because the flow rate $\mathrm{Q}$ and the resistance to flow are functions of the curvature $\mathrm{H}$, as indicated in eqn. (23). If the electric field $\mathrm{E}$ is constant in eqn. (24), we recuperate the static flexoelectric result $[7,8]$ :

$$
\mathrm{H}_{\mathrm{o}}=\frac{\mathrm{E}_{\mathrm{o}} \mathbb{C I}}{2\left(\gamma_{\mathrm{o}}+\left(2 \mathrm{k}_{\mathrm{c}}+\overline{\mathrm{k}}_{\mathrm{c}}\right) \frac{\mathbb{C}}{2}\right)}
$$

which indicates that curvature is the ratio of driving force $\left(\mathrm{E}_{\mathrm{o}} \mathbb{C} \mathfrak{I}\right)$ to resistance $\left(2 \gamma_{\mathrm{o}}+\left(2 \mathrm{k}_{\mathrm{c}}+\overline{\mathrm{k}}_{\mathrm{c}}\right) \mathbb{C}\right)$. Again, if we neglect tension and torsion, eqn. (25) is consistent with eqn. (1).

\section{FREQUENCY RESPONSE OF DRIVEN FLEXOELECTRIC MEMBRANES}

Next, we analyze the response of the actuator system to an externally imposed sinusoidal electric field given by: $\mathrm{E}(\mathrm{t})=\mathrm{E}_{\mathrm{o}} \sin \omega \mathrm{t}$. It proves useful to nondimensionalize the governing eqn. (24). Scaling the electric field with the $\mathbb{C} \mathfrak{a} / 2 \gamma_{0}$, the time with driving frequency $\omega$, and the curvature $\mathrm{H}$ with the capillary diameter "a," eqn. (24) becomes:

$$
\overline{\mathrm{E}}_{\mathrm{o}} \sin \overline{\mathrm{t}}=\operatorname{De} \overline{\mathrm{H}} \frac{\mathrm{d} \overline{\mathrm{H}}}{\mathrm{d} \overline{\mathrm{t}}}+\left(1+\left(2 \mathrm{k}_{\mathrm{c}}+\overline{\mathrm{k}}_{\mathrm{c}}\right) \frac{\mathfrak{I}}{2 \gamma_{\mathrm{o}}}\right) \overline{\mathrm{H}}
$$


where the Deborah number De and resonant frequency $\omega_{\mathrm{o}}$ are:

$$
\mathrm{De}=\frac{\omega}{\omega_{0}} ; \quad \omega_{\mathrm{o}}=\frac{\gamma_{\mathrm{o}}}{\eta \mathrm{a}}
$$

The Deborah number is the ratio of the imposed frequency $\omega$ to the resonant frequency $\omega_{0}$. The magnitude of the De number defines the viscoelastic response of the system and hence we seek a series solution of the form:

$$
\overline{\mathrm{H}}=\overline{\mathrm{H}}_{\mathrm{o}}+\mathrm{De}_{\overline{\mathrm{H}}}+\mathrm{De}^{2} \overline{\mathrm{H}}_{2}+\cdots
$$

Substituting the expansion (28) into eqn. (26), we get:

$$
\begin{gathered}
\overline{\mathrm{E}}_{\mathrm{o}} \sin \overline{\mathrm{t}}=\operatorname{De}\left(\overline{\mathrm{H}}_{\mathrm{o}}+\operatorname{De} \overline{\mathrm{H}}_{1}+\cdots\right) \frac{\mathrm{d}\left(\overline{\mathrm{H}}_{\mathrm{o}}+\operatorname{De} \overline{\mathrm{H}}_{1}+\cdots\right)}{\mathrm{d} \overline{\mathrm{t}}}+\mathbb{R}\left(\overline{\mathrm{H}}_{\mathrm{o}}+\operatorname{De}_{1}+\cdots\right) \\
\mathbb{R}=1+\left(2 \mathrm{k}_{\mathrm{c}}+\overline{\mathrm{k}}_{\mathrm{c}}\right) \frac{\mathfrak{I}}{2 \gamma_{\mathrm{o}}}
\end{gathered}
$$

The curvature response, obtained by solving eqn. (29) at each order of the Deborah number De, is given by:

$$
\overline{\mathrm{H}}=\frac{\overline{\mathrm{E}}_{\mathrm{o}}}{\mathbb{R}} \sin \overline{\mathrm{t}}-\frac{D \overline{\mathrm{E}}_{\mathrm{o}}^{2}}{(2 \mathbb{R})^{3}} \sin (2 \overline{\mathrm{t}})+\cdots+\frac{(-1)^{\mathrm{n}} 2 \cdots \times 2^{\mathrm{n}-2} \times 2^{\mathrm{n}-1} \operatorname{De}^{\mathrm{n}} \overline{\mathrm{E}}_{\mathrm{o}}^{2^{\mathrm{n}}}}{(2 \mathbb{R})^{2^{\mathrm{n}}-1}} \sin \left(2^{\mathrm{n}} \overline{\mathrm{t}}\right)+\cdots
$$

where the first term denotes a purely elastic response and the remainder contains the viscoelastic response in terms of higher harmonics of even order. Next, we discuss the main signatures of the response: (i) the anharmonicty, (ii) the zero frequency response, and (iii) the resonance frequencies, as follows:

1. The membrane response to fluctuating electric field is anharmonic and involves even harmonics: $\sin \omega t, \sin 2 \omega t, \sin 4 \omega t, \ldots, \sin 2^{\mathrm{n}} \omega \mathrm{t}, \ldots$. The origin of the anharmonicity is the viscous dissipation term in eqn. (26) that arises, as mentioned above, because both the flow rate and resistance in the capillary Poiseuille flow are functions of the average curvature $\mathrm{H}$.

2. According to eqn. (31) the zero frequency limit, $\omega \rightarrow 0$, is purely elastic and given by:

$$
\mathrm{H}(\omega \rightarrow 0, \mathrm{t})=\mathrm{H}_{\mathrm{s}} \sin \omega \mathrm{t} ; \quad \mathrm{H}_{\mathrm{s}}=\frac{\mathrm{E}_{\mathrm{o}} \mathbb{C I}}{2 \gamma_{\mathrm{o}}+\left(2 \mathrm{k}_{\mathrm{c}}+\overline{\mathrm{k}}_{\mathrm{c}}\right) \mathfrak{I}}
$$

Equation (32) agrees with the expected steady state response given in eqn. (25). Increasing the field increases the curvature amplitude. As an example of the application of eqn. (32), for typical values quoted in [2]: $\mathrm{E}_{\mathrm{o}}=25 \mathrm{mV} / \mathrm{nm}, \gamma_{\mathrm{o}}=5 \mathrm{mN} / \mathrm{m}, \mathrm{a}=0.5 \mathrm{~mm}, \mathbb{C}=20 \times 10^{-18} \mathrm{C}, \mathrm{k}_{\mathrm{c}}=\overline{\mathrm{k}}_{\mathrm{c}}=0$, it is found that $\mathrm{R} \approx 0.5 \mathrm{~m}$. At low driving frequencies, the membranes oscillate with the field and the coefficients of the higher harmonics in eqn. (31) are negligible;

3. The present nonlinear model (eqn. (26)) predicts sub-harmonic resonances. Hence scanning the frequency $\omega$ we expect to encounter secondary resonance peaks at $2 \omega=\omega_{0}$, $4 \omega=\omega_{0}, \ldots, 2^{\mathrm{n}} \omega=\omega_{0}, \ldots$. The presence of these secondary resonances is due to the dissipative nonlinearity. 
Electromechanical models of the outer hair cell involved in mammalian hearing have been recently reviewed [15]; it is concluded that a significant number of fundamental issues need to be better understood and that modeling will continue to be a major driving force in understanding hearing. In this respect, the response of the outer hair cells to high frequency conditions and the cell viscosity continue to pose fundamental questions [15]. In the present paper, we have computed the frequency response of a simple flexoelectric [16] membrane taking into account the viscosity of the surrounding phases, founding resonant frequencies without appeal to strain energies. This finding proves that flexoelectricity has a role to play in more generalized models of outer hair cells.

\section{CONCLUSIONS}

This paper presents a model for the response of flexoelectric membranes to external oscillating electric fields, in contact with viscous phases, relevant to biological processes such as the outer hair cells in the hearing apparatus. The membrane-viscous fluid system acts as an actuator in the presence of the electric field, creating membrane curvature as well as viscous flow in the adjoining phases. The viscous dissipation in the adjoining phases gives rises to a nonlinearity in the actuator system. The frequency response of the viscoleastic actuator to an oscillating electric field is computed using a series expansion in terms of the Deborah number. The actuator anharmonic response is given in terms of a fundamental and even harmonics. The importance of the even harmonics increases with increasing Deborah number, or ratio between the driving frequency $\omega$ and the resonant frequency $\omega_{0}$. As expected in driven nonlinear systems, subsidiary resonant peaks appear at driving frequencies that are equal to $\omega_{0} / 2^{n} ; n=1,2,3, \ldots$, where $1 / \omega_{\mathrm{o}}$ is the relaxation time of the actuator.

The present results contribute to the on-going work on mechano-transduction in biological systems and to the current work on applications of flexoelectricity to hearing processes [17]. The basic actuator flexoelectric mechanism was used to describe the role of electromotility in the process of mechanoamplification that supports the detection of high frequency sounds by outer hair cells. In this model [16], nanoscale curvature of the plasma membrane of outer hair cells changes by depolarization or polarization. This change in curvature is transmitted to the underlying cytoskeleton causing length changes that amplify vibrations. Since membranes participating in the hearing process are in contact with viscous and/or viscoelastic phases and subjected to multiple frequencies, the nonlinearities in the actuator model predict that combination frequencies are likely to occur [9]. In addition, the viscous nonlinearity in conjunction with high amplitude oscillating electric field has the potential to exhibit chaotic responses. Future work will extend the present work to oscillating membranes immersed in viscoelastic media.

\section{ACKNOWLEDGEMENT}

This research was supported by the Natural Science and Engineering Council of Canada.

\section{REFERENCES}

[1] Wallmersperger, T., Kroplin, B. \& Gulch, R.W., Polyelectrolyte gels. Chemo-Mechanical Couplings in Porous Media Geomechanics and Biomechanics, eds B. Loret \& J.M. Huyghe, Springer Wien: New York, pp. 333 - 382, 2004.

[2] Petrov, A.G., The Lyotropic State of Matter, Gordon and Breach Science Publishers: Amsterdam, 1999.

[3] Todorov, A.T., Petrov, G. \& Fendler, J.H., First observation of the converse flexoelectric effect in bilayer lipid membranes. J. Phys. Chem., 98, pp. 3076 - 3079, 1994.

[4] Petrov, A.G., Dynamics and Defects in Liquid Crystals, eds P.E. Cladis \& P. Palffy-Muhoray, Gordon and Breach Publishers: Amsterdam, pp. 255 - 262, 1998. 
[5] Edwards, D.A., Brenner, H. \& Wasan, D.T., Interfacial Transport Processes and Rheology, Butterworth: MA, 1991.

[6] Slattery, J.C., Sagis, L. \& Oh, E.-S., Interfacial Transport Phenomena, 2nd edn, Springer Verlag: New York, 2007.

[7] Rey, A.D. Capillary models for liquid crystal fibers, membranes, films, and drops. Soft Matter, 2, pp. $1349-1368,2007$.

[8] Rey, A.D., A flexoelectric membrane model. Physical Review E, 74(1-1), 011710/1011710/11, 2006.

[9] Main, I.G., Vibrations and Waves in Physics, 3rd edn, Cambridge University Press: Cambridge, 1993.

[10] Panagopoulos, D.J., Karabarbounis, A. \& Margaritis, L.H., Mechanism for action of electromagnetic fields on cells. Biochemical and Biophysical Research Communications, 298, pp. 95-102, 2002.

[11] Panagopoulos, D.J., Messini, N., Karabarbounis, A., Philippetis, A.L. \& Margaritis, L.H., A mechanism for action of oscillating electric fields on cells. Biochemical and Biophysical Research Communications, 272, pp. 634-640, 2000.

[12] Kralchevsky, P.A. \& Nagayama, K., Particles at Fluids Interfaces and Membranes, Elsevier: Amsterdam, 2001.

[13] Bird, R.B., Armstrong, R.C. \& Hassager, O., Dynamics of Polymeric Liquids, Volume 1, John Wiley: New York, 1987.

[14] Yucel, U. \& Kazakia, J.Y., Viscoelastic effects in pressure transduction. J. Non-Newtonian Fluid Mech., 123, pp. 59-66, 2004.

[15] Spector, A.A., Deo, N., Ratnanather, J.T. \& Raphael, R.M., Electromechanical models of the outer hair cell composite membrane. J. Membrane Biol., 209, pp. 135-152, 2006.

[16] Petrov, A.G., Electricity and mechanics of biomembrane systems: flexoelectricity in living membranes. Analytica Chimica Acta, 568, 70-83, 2006.

[17] Raphael, R.M., Popel, A.S. \& Brownell, W.E., A membrane bending model of outer hair cell electromotility. Biophys. J., 78, pp. 2844-2862, 2000. 